\title{
Evidence-Based Practice Nursing Interventions for Improved Functional and Cognitive Outcomes in the Traumatic Brain Injury Patient
} Judith Kutzleb ${ }^{1,2 *}$

${ }^{1}$ Assistant Professor of Nursing, School of Nursing and Allied Health, Fairleigh Dickinson University, Teaneck, New Jersey, USA ${ }^{2}$ Doctor of Nursing Practice - Adult Medical Clinic at Holy Name Medical Center, Teaneck, New Jersey, USA

\begin{abstract}
Problem: Traumatic Brain Injury (TBI) results in a combination of physical, cognitive, and behavioral impairments with an estimated 2 million Americans sustaining TBI every year. The overall aim of this program was to implement evidence-based practice protocols for clinical management of traumatic brain injury patients. The routine integration of the following Evidence-Based Practice protocols: pulse oximetry and blood pressure monitoring every two hours, toileting and limited distance ambulation (10 to 25 feet) every two hours during the patients wakeful state, baseline Orientation-Log assessment (O-Log) on admission then on a daily basis, physical therapy and speech/cognitive therapy evaluations within 24 to 48 hours of admission were implemented to improve the functional and cognitive outcomes, and reduce bedside patient sitters in the acute care setting.
\end{abstract}

Data source: This was an exploratory pilot program that implemented EBP protocols for the clinical management of TBI patients. An analysis of trends (pre-EBP of 58 TBI patients vs. post-EBP of 50 TBI patients) was utilized to evaluate whether the change in practice made a significant difference in improving patient's outcome.

Conclusion: The EBP protocols decreased sitter sessions by $80 \%$ and enabled TBI patients to achieve states of functional and cognitive well-being with a structured approach to clinical management. The finding for sitter session usage showed a reduction from 30 sessions 3 months before program implementation to 6 sessions during program implementation, with a continued sitter session reduction of 0 sessions for 6 months post program implementation. The results of this program established a structured and sustainable approach to the clinical management of TBI patients. Through the strategic cycle of patient assessment, ambulation, toileting and hemodynamic status evaluation, patients became less apt to develop confused and agitated states, which supported a safer patient environment and reduced the need for sitter sessions.

Implications for Practice: Results indicate that the EBP protocol created a structured approach to clinical patient management for the nursing staff. The continuous repetition of patient interventions supported by the protocols in concert with staff education on TBI and its consequences, created skill development in the nursing staff for assessing and managing altered states in this patient population.

Keywords: Traumatic brain injury; Evidence-based guidelines; Treatment; Rehabilitation; Prognosis

\section{Introduction}

Traumatic brain injury (TBI) results in a combination of physical, cognitive, and behavioral impairments. Broadly defined, traumatic brain injury is incurred due to externally inflicted trauma, which in turn results in impairment of an individual's physical, cognitive and psychosocial functioning. Every year, an estimated 2 million Americans sustain a traumatic brain injury [1], which principally results from vehicular accidents, falls, acts of violence and sports injuries. Traumatic brain injury exceeds the combined incidence of breast cancer, human immunodeficiency virus, spinal cord injury and multiple sclerosis [1]. The risk of traumatic brain injury is highest in the adolescent and young adult population between the ages of 15 to 24 years of age, and in the aged population 75 years and older [1]. The National Institute of Neurologic Disorders and Stroke statistics clearly identified males being 1.5 times more likely than females to suffer a traumatic brain injury [1]. An alarming number of affected individuals, according to Centers for Disease Control and Prevention, are the 5.3 million Americans who are currently impaired from traumatic brain injury, thus making it one of the most common disabling conditions [2].

\section{Significance of the Problem}

The consequences of traumatic brain injury include a significant change in the individual's life course, overwhelming disruption of family dynamics, massive loss of income or earning potential and costly lifetime expenses. Hospital admissions are approximately 300,000 annually for persons with mild or moderate traumatic brain injury, with an additional unknown number that go undiagnosed [1] Traumatic brain injury presents with serious long-term cognitive and behavioral problems that effectively prevent injured individuals from fulfilling their previous societal roles. This becomes a more problematic consequence because it involves an individual's cognition, emotional function and behavior, which ultimately affects interpersonal relationships, school, work and personal safety. The impact of this injury on patient's cognitive and behavioral functions poses many challenges for nursing management in the hospital setting. Integrating evidence-based practice approaches to traumatic brain injury is critical in optimizing an individual's safety, recovery and functional ability in the acute care environment.

In the past several years there has been an unprecedented interest in patient safety and the quality of health care. To address the pitfalls in the American health care system, The Committee on the Quality of Health Care in America was formed in June 1998 to develop a strategy to substantially improve the quality of health care. In 2001, the committee

${ }^{*}$ Corresponding author: Judith Kutzleb, 49 Franklin Avenue, Hawthorne, New Jersey, 07506, USA, Tel: (973) 600-5991; E-mail: kutzlebd@aol.com

Received March 12, 2012; Accepted April 11, 2012; Published April 13, 2012

Citation: Kutzleb J (2012) Evidence-Based Practice Nursing Interventions for Improved Functional and Cognitive Outcomes in the Traumatic Brain Injury Patient. J Nurs Care 1:110. doi:10.4172/2167-1168.1000110

Copyright: (ㅇ 2012 Kutzleb J. This is an open-access article distributed under the terms of the Creative Commons Attribution License, which permits unrestricted use, distribution, and reproduction in any medium, provided the original author and source are credited. 
addressed the urgent quality problem of patient safety. The report of the Institute of Medicine titled, "To Err is Human: Building a Safer Health System" [3] presented the central message that tens of thousands of Americans die each year from errors in their care. The patient safety report was a call for action to make care safer, and also called for action to improve the American health care system as a whole. Patient safety is now a national priority, and innovative programs to address and improve patient safety are currently a health care priority.

The development of evidence-based protocols evolved from a lack of standardized clinical management in the traumatic brain injury patient population. Secondary trauma from patient falls is a significant adverse event in the acute care setting. As a proactive measure to ensure patient safety, a patient sitter program was developed through the department of nursing at a University Medical Center in Northern New Jersey. Patient sitters are defined as non-medical, non-nursing hospital employees, who have a job responsibility of sitting at a hospitalized patient's bedside. The hospitalized patients for which patient sitters are used have been defined by the organization and include patients with suicidal ideation awaiting an in-patient bed on psychiatry, patients who are agitated and unable to make self-care decisions, and patients who are confused to person, place and time, displaying active behavior deemed harmful to themselves. The impetus for this patient safety initiative was due to the continuing rise in the number of patient sitter sessions without a reduction in secondary trauma events, thus creating a costly burden for the institution. All TBI patients were admitted to the intermediate care unit which had a greater than two-fold monthly increase of sitter sessions from 18 to 46 during the months preceding program implementation. Hence, improved patient outcomes and sitter session reduction became an administrative driven priority.

Traumatic brain injury as defined by the Mild Traumatic Brain Injury Committee of the Head Injury Interdisciplinary Special Interest Group of the American Congress of Rehabilitation Medicine is: any patient with mild traumatic brain injury is a person who has a traumatically induced physiological disruption of brain function, which is manifested by at least one of the following: any period of loss of consciousness, any loss of memory for events immediately before or after the accident, any alteration in mental state at the time of the accident (e.g., feeling dazed, disoriented, or confused), and focal neurological deficit(s) that may not be transient, but where the severity of the injury does not exceed a period of a loss of consciousness that is approximately 30 minutes or less, and after 30 minutes, the initial Glasgow Coma Scale is 1315, and posttraumatic amnesia is not greater than 24 hours [4]. This landmark operational definition of TBI is used to define the population enrolled in this program. Objective measures to assess outcomes in the TBI patient population were formulated from quantitative and metaanalyses studies.

In concert with the hospital performance improvement initiative for sitter session reduction, the focus of this program was through a three pronged approach: first to evaluate staff education on evidencebased practice concepts and application with traumatic brain injury patient management, second, to evaluate the extent to which staff education for implementation of evidence-based protocols influences overall patient management with reduction in sitter usage, and third, to target efforts to improve patient safety and patient outcomes in this patient population. The impetus to successful program initiation is formulating a clinical question. The clinical question addressed by this program was: In traumatic brain injured patients (TBI) 22 years of age and older in the acute care setting, does the implementation of TBI patient management guidelines reduce patient sitter usage by $50 \%$ compared to usual patient management.

\section{PICO}

The population consisted of 50 patients 22 years of age and older admitted to the intermediate care unit with a recent history of traumatic brain injury. The intervention included: pulse oximetry monitoring, blood pressure monitoring, memory assessment evaluation, toileting and limited distance ambulation. The Orientation-log (O-Log) developed by Dr. Thomas Novack was the tool completed by the primary nurse at the time of admission, then daily until discharge and measured the patient's orientation in terms of place, time and situation. The O-Log provided a standardized guideline for performing consistent orientation assessments in the TBI patient population. Staff education consisted of three phases. Phase I was the dissemination of evidencebased practice concepts and practice implementation to enhance the nursing staff's understanding of identifying and answering a clinically important question which requires evidence-based solutions. Phase II consisted of educational content on differentiating types of traumatic brain injury (concussion, contusion, subarachnoid, subdural, and epidural hemorrhages), etiology of TBI, population involved, and patient management. Phase III incorporated the educational content on evidence-based protocols for TBI patient management developed from best practice extrapolated from the literature. Each educational phase included a comprehensive topic overview and case study analysis for all staff on all shifts. The comparison of interest population was that of usual TBI patient management. The outcome is to reduce patient sitters during the hospital admission by $50 \%$.

\section{Review of the Literature}

A literature search was done based on the population and identified problems. When sufficient research was found as a basis to implement practice change, an exploratory pilot program was designed. Evidence-based practice guidelines were then designed for program implementation and staff education on evidence-based practice concepts, traumatic brain injury patient management and program implementation was initiated. Once the change in practice for TBI patient management was identified and agreed upon by organizational management and staff as "appropriate for adoption into practice", the implementation phase of evidence based practice protocols for traumatic brain injury patients was initiated.

A systematic search of the literature on traumatic brain injury used the following databases: MEDLINE (1997-2008), CINHAL (1997-2008) and EMBASE (1997-2008). Articles were considered relevant if they addressed diagnosis, incidence, risk factors, treatment and rehabilitation, prognosis and prevention. Inclusion criteria were randomized controlled trials, large cohort and case-controlled studies. Studies on penetrating brain injuries, shaken-baby syndrome, stroke, and studies using non-human subjects, cadavers, crash-test dummies or biomechanical simulation were excluded. Two of the five LOE I studies which had the strongest correlation to effective acute care TBI program implementation were the randomized controlled trials by Chapman and Wade.

Chapman et al. in the study "Homeopathic treatment of mild traumatic brain injury: a randomized clinical trial" was a Randomized Control Trial (RCT) $(\mathrm{n}=61)$ and compared homeopathic treatment $(n=32)$ with placebo $(n=29)$ during a four month period. The active treatment group had a significantly better Functional Independence Measure Score (FIM) cognitive total score compared to placebo ( $\mathrm{P}$ $=0.257)$. The active treatment group also showed improvement over the placebo group on scales measuring activities of daily living ( $\mathrm{P}$ $<0.036)$. Results suggested both groups improved, but the homeopathic 
treatment group had a significant reduction in cognitive symptoms and disability [5].

Wade et al. compared standard hospital care with intervention patients who also received early follow-up 7-10 days post injury ( $\mathrm{n}=$ 1156). Only patients who were admitted to the hospital were included in the study. Those patients receiving the early follow-up service through the head injury service had better outcomes in cognition $(\mathrm{P}<0.001)$ and complete remission of depression $(\mathrm{P}<0.001)$ than the usual care group. The intervention group had significantly less social disability and lower ratings of post-concussion symptoms at 6-months after the injury than the control group [6].

One LOE II study by Cifu et al. did a multicenter analysis on the relationship between therapy intensity and rehabilitation outcomes after traumatic brain injury. The objective was to identify factors relating to the intensity of rehabilitation services received and to ascertain the relation between injury outcomes, demographics, types of therapy, and the intensity of rehabilitation services provided. There were 491 enrolled patients with a mean age of \pm SD $34.3 \pm 15.88$ years in a setting where continuum of care included emergency medical services, intensive and acute care medical care and inpatient rehabilitation. The results showed that age predicted the intensity of both psychologic $(\mathrm{P}<0.001)$ and total therapy $(\mathrm{P}<0.01)$ services. Acute care length of stay was also a significant predictor of psychologic/cognitive services $(\mathrm{P}<0.01)$. Therapy intensity was predictive of motor functioning at discharge $(\mathrm{P}<$ 0.001). Findings support the assertions that increased therapy intensity, particularly physical and psychologic/cognitive therapies, enhances functional outcomes [7].

One Level III study found in the literature focused on initiating intensive rehabilitation during the in-patient hospital admission. Shiel et al. focused on the effect of increased in tensity of rehabilitation therapy provided to brain injured subjects, the rate at which independence was regained, and the duration of hospital admission. The design was a twoyear prospective multicenter controlled study of fifty-six individuals with moderate and severe head injury. The results of the therapy interventions supported the recommendations that the subjects who received more intensive therapy made more rapid progress and were discharged sooner from the hospital. This study also supported that increasing the hours per week of therapy given to adults recovering from brain injury in-hospital, can accelerate the rate of recovery of personal independence and thus result in their being discharged from the hospital sooner [8].

One Level VII study extrapolated from the literature by D.W. Marion supported the implementation of evidence-based practice in TBI management. The publication focused on the effects of evidencebased guidelines in the management and outcomes of traumatic brain injuries from pre-hospital to hospital admission which included close monitoring of oxygenation and blood pressure, Glasgow Comma Score (GCS), fluid resuscitation and airway, ventilation and oxygenation [9]. The diagnostic and prognostic value for oxygenation and blood pressure monitoring on a continual basis had been suggestive that hypoxemia and hypotension is associated with poor outcomes. The GCS is a significant and reliable indicator of the severity of TBI particularly in association with repeated scoring and improvement or deterioration of the score over time. Fluid resuscitation is essential to avoid hypotension and or limit hypotension to the shortest duration possible. Studies support the maintenance of a normotensive state to promote circulation to the essential organs to promote optimal brain recovery. The rationale for the development and implementation of evidence-based guidelines in TBI care is two-fold: first, that there are best practices, and second, that consistent care in treating and managing TBI patients will lead to improved outcomes. This research concluded that consistent protocoldriven care helps to establish a team approach, eliminates confusion and eliminates secondary trauma leading to improved outcomes in physical and cognitive function [9].

\section{Instrument}

The Orientation-Log (O-Log) developed by Dr. Thomas A Novack was the instrument used in this program. The O-Log is an assessment instrument that measures orientation in terms of place, time and situation in the TBI patient population. Mental confusion can be a significant barrier to independent living and participation in rehabilitation. Measuring orientation is a way to evaluate confusion and is useful in documenting change over time. Orientation assessment is a component of all measures of mental status largely because it is quick to ascertain, can be scored objectively, and is generally agreed to be within the cognitive capacity of normal individuals [10-11]. The objective of the instrument is to introduce a brief quantitative measure of cognitive orientation. It has been developed for daily use at the inpatient bedside. The O-Log is a 10-item scale which is formatted for rapid visual analysis or orientation trends that can be used to evaluate pharmacologic and cognitive-behavioral interventions. The ten items of the O-log are scored 0-3 depending on the response provided. Scores for each of the ten items are summed to obtain a total score that ranges from 0 to 30 . A score of greater than 25 on two consecutive assessments signals the beginning of continuous day-to-day memory, and is the most important index of TBI recovery [10-11]. The O-log's reliability (interrater and internal consistency) has been measured across fifteen neurorehabilitation centers. It is the standard instrument used across the country to measure cognition in TBI patients. For individual items, a Spearman rho interrater reliability coefficient ranges from 0.851 to 1.00. The interrater reliability to the total score was 0.993 . The O-Log internal consistency (coefficient alpha) was 0.992 .

\section{Methodology and Implementation}

The evidence-based practice model used in this program was the Iowa Model of Evidence-Based Practice, developed by Marita G Titler [12]. The Iowa Model is an organizational model incorporating the conduct and use of research and other forms of evidence such as theories, expert opinions and case reports to design a program that addresses either a problem focused trigger or knowledge focused trigger to improve outcomes. If change in current practice is warranted, the changes are implemented using a process of planned change which includes a practice pilot tested with a small group of clients. Guidelines are then revised based on the pilot. Outcome data is collected, and decision points regarding the problem are clearly identified according to organizational priority. Sufficient research is gleaned to form a basis for change, and whether the proposed change is appropriate for adoption into practice.

The Iowa Model melds quality improvement with research utilization in an algorithm that nurses find intuitively understandable. Unique to the Iowa Model is the concept of triggers of evidence-based practice. Evidence-based practice may be spurred by a clinical problem or by knowledge coming from outside an organization. Either of these triggers can set an evidence-based practice project into motion. The Iowa Model for this program stemmed from a problem focused trigger identified in the performance improvement data regarding increased patient sitter usage. Once this problem was identified as an organizational priority an evidence-based practice team was formed. The team consisted of nursing management, nursing staff (clinical, 
secretarial, and ancillary), case management and social service members. Unit selection was based on sitter usage volume, and TBI patient population was selected based on both admission volume and patient sitter utilization.

This program employed an evidence-based protocol for TBI patient management, identified in the evidence-based literature suggesting that early and intensive cognitive and physical therapeutic interventions, in concert with focused nursing interventions, would reduce the number of patient sitters, improve functional patient outcomes, and reduce states of agitation and confusion in the TBI patients.

The sample population consisted of 50 patients 22 years of age or older admitted to the intermediate care unit as either a direct admission from the Emergency Trauma Department or as a transfer from the surgical intensive care unit with a recent history of traumatic brain injury.

Exclusion criteria included those patients who presented with traumatic brain injury in the setting of either an ischemic or hemorrhagic stroke, brain mass or lesion, as well as those patients where traumatic brain injury was not thought to be the primary problem. Patients with illness that could compromise survival over the duration of the study as well as patients taking mood-altering medications (antipsychotics and or anxiolitics) prior to admission, and patients with alcohol abuse prior to admission to the hospital were excluded.

The TBI diagnosis was based on diagnostic results of either a computed tomography scan or a magnetic resonance imaging scan, mechanism of injury with either an associated loss of consciousness, altered mental status, behavioral or mood change in all patients 22 years of age and older by the trauma surgeon. The primary care nurse and the nursing assistant and/or ancillary staff member had defined responsibilities in the implementation of the evidence-based protocol for TBI patient management. The unit selection was based on volume of patient sitter usage and TBI admissions. Prior to program implementation, there were a total of $58 \mathrm{TBI}$ patients admitted to the intermediate care unit. Of the 58 patients, a total of 30 sitter sessions were utilized. The number of sitter sessions was obtained from the nursing department staffing statistics. The intermediate care unit holds a capacity of 24 patients, of which 6-7 beds are occupied by TBI patients at any given time. Program implementation followed the Iowa Model, and the patients were managed according to the evidence-based protocols designed for this program. The protocol followed by the primary nurse and the ancillary staff consisted of:

- Daily cognitive evaluations by the primary nurse with every two hour vital signs consisting of blood pressure and pulse oximetry by the ancillary staff. Increasing nursing awareness of patient orientation enabled the nursing staff to quickly identify changes in patient status while providing more direct patient care.

- Patient orientation was assessed in terms of place, time, and situation on admission by the primary care nurse. The orientation $\log (\mathrm{O}-\mathrm{Log})$ was used by the nursing staff as a guideline for performing consistent orientation assessments.

- Physical therapy order was entered by the primary nurse on admission for the consult to be completed within 24-hours. Once the initial consult was completed, physical therapy continued twice daily until discharge. Once the physical therapy evaluation was completed, toileting and limited distance ambulation (minimally 10 feet to maximally 25 feet) was done every two hours by the ancillary staff while the patient was in a wakeful state.
- Speech/cognitive order was entered by the primary nurse on admission for the consult to be completed within 24-hours. Once the initial speech/cognitive evaluation is completed therapy was continued three times per week until discharge.

- The primary nurse initiated a case management and social service referral within 24-hours of admission for appropriate discharge planning.

Based on review of the literature, the best evidence showed that a staff education program consisting of TBI patient management and clinical implementation of evidence-based guidelines will improve outcomes and reduce the use of patient sitter sessions by $50 \%$. Staff education on evidence-based practice protocols in traumatic brain injury management was provided to all staff on all shifts prior to the beginning of the program. TBI protocol implementation was monitored throughout the duration of the program. The pilot program was extended for three months, with permission for this evidencebased program granted by the Institutional Review Board.

The goal for implementation of this evidence-based exploratory pilot program was to integrate routine use of evidence-based practice in TBI patient management. Deliberate program planning and preparatory work became the foundation for an evidence-based practice philosophy of care.

An implementation process was developed and followed throughout the EBP project. Program success is dependent on three essential components: developing a sturdy foundation, inclusion of support structures and maintaining a focus to fine details [12]. The foundation for program implementation was based on organizational support and recognition for a need in changing clinical practice in the nursing management of TBI patients. Project champions were selected by the nurse manager based on individual staff interest. Educational staff in-services were planned and conducted on all shifts.

To maintain an open line of communication regarding program implementation timelines, weekly informational flyers were posted. Open forums to discuss all questions regarding TBI patient admission processes, utilization of the O-log, patient assessment form, computer order entry for physical therapy, speech/cognitive therapy consults and problems encountered with program implementation were scheduled. This afforded one-on-one conversations with staff members to provide opportunities for TBI protocol clarification and problem-solving. It also provided the opportunity for the staff to ask questions about evidencebased practice and to recognize the role of evidence-based practice in clinical practice decisions.

The O-Log's structured sequence of questions was a guideline for consistent orientation assessments in the TBI patient by the nursing staff. This educational session also included the role of close monitoring of vital signs, neurological evaluations, toileting and ambulation, and the impact that these interventions have on outcomes as supported by the literature. Lastly, the staff was educated on how the timely notification of ancillary services for physical therapy, speech/cognitive therapy for consult and initial patient evaluation, and case management and social service impacted in the planning of therapeutic interventions best suited for the individual patient and initiating essential patient screenings in order to establish a comprehensive discharge plan.

Consistent attention to staff performance with protocol implementation, staff support and encouragement with protocol implementation, and clarification of any and all questions during the initial stages was integral in order to promote a seamless implementation of this program. It also ensured that clinical practice based on evidence 
became a daily mindset for every clinician. Supportive structures were created through designing EBP workgroups comprised of staff champions.

Through this workgroup of staff champions a TBI patient admission packet was designed which consisted of a protocol check list, the O-Log form, and the two-hour patient assessment form. This packet standardized the admission paperwork for all TBI patients admitted to the intermediate care unit. Practice standards and documentation guidelines were also designed in concert with the best evidence gleaned from the literature. Worksheets were also designed by the workgroup of staff champions in order to disseminate information during shift report as well as to ensure all TBI protocols were consistently followed. The work sheets contained a checklist for ancillary department consults to ensure all consults were requested at time of patient admission. Weekly meetings were scheduled as a conduit to address any and all real-time clinical issues that might have developed during program implementation. The workgroup also provided 24-hour access to clinical information by staff, by ensuring a workgroup member was scheduled on every shift on a daily basis. An evidence-based practice reference module was also available to all staff on the unit throughout the program implementation timeframe. The TBI protocol implementation was monitored on an on-going basis with each admission. This program created autonomy in the workgroup champions to review each TBI admission so as to track the adherence to protocol implementation and monitor program outcomes within the designated timeframes.

Progress on program implementation of the protocol was shared at monthly unit staff meetings as well as at the monthly evidence-based practice council meetings. Progress reports consisted of a review of each TBI admission with a review of each patient admission packet which included the protocol check list, the O-Log form, and the twohour patient assessment form, to monitor adherence. A combination of staff education, continual protocol monitoring, and multidisciplinary interventions supported the evolving clinical practice change.

\section{Results and Recommendations}

Of the 50 patients in the TBI project, $70 \%$ (thirty-five) were males and $30 \%$ (fifteen) were females. Six sitter sessions were utilized during the three months of program implementation for mild TBI patients with a Glasgow Coma Scale (GCS) of 13 to 15. This result was compared to the comparative TBI population with a GCS of 13 to 15 for the three months preceding program implementation, where there were 30 sitter sessions utilized for 58 TBI patients (Figure 1).

The daily cognitive evaluations by the nursing staff were completed during the morning initial patient assessment on a daily basis throughout program implementation. The use of the O-Log by the nursing staff provided a consistent guideline of questions to assess the level of patient orientation. The cognitive evaluation consisted of place, time and circumstance. Place was evaluated by asking the patient for the name of the city and the hospital. Time was evaluated by asking for the name of the month, the date, the year, the day of the week, and the time of day. Circumstance was evaluated by asking the patient to recall what event occurred, and to describe what injury occurred. The cognitive evaluations were maintained on a daily basis by the nursing staff from time of patient admission to day of hospital discharge.

Vital signs were obtained by the ancillary staff every two hours and documented on the patient assessment form during a wakeful state. The wakeful state was defined by the workgroup champions as being between the hours of $6 \mathrm{am}$ to $11 \mathrm{pm}$. The nursing staff reviewed the assessment form from the ancillary staff member prior to the

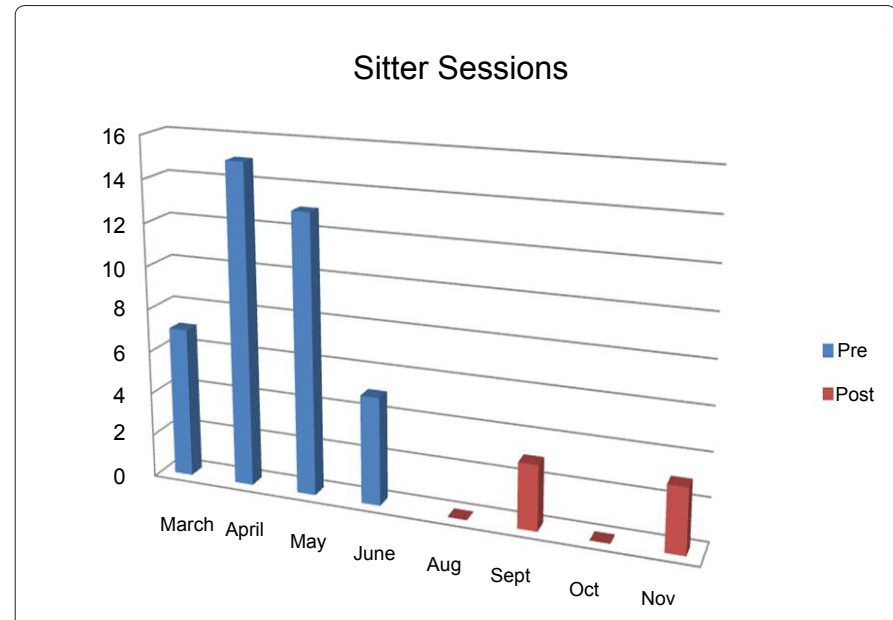

Figure 1: Sitter Sessions

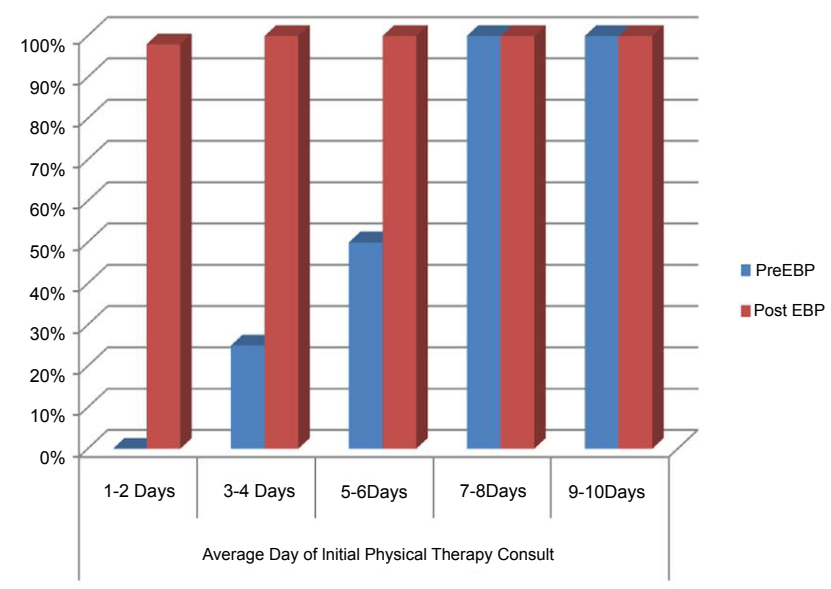

Figure 2: Physical Therapy Consults

transcription of the vital signs into the permanent patient record.

Physical therapy consult evaluations progressively increased to $100 \%$ within the first 24 to 48 hours of admission with the implementation of the TBI protocol (Figure 2). Patients received an intensive and comprehensive physical therapy program on a consistent basis during their hospital admission. The intensive physical therapy program provided the opportunity for patients to gait train and ambulate twice daily in the physical therapy department, as well as on an every two-hour basis on the unit, which enhanced physical strength and independence and promoted regulation of physiologic balance and sleep-wake cycles.

Speech/Cognitive therapy consult evaluations increased to $100 \%$ by 48 hours after each TBI admission (Figure 3). Prior to program implementation, speech/cognitive consults were only rendered to those patients whose planned discharges were to an acute rehabilitation facility, which consisted of only $25 \%$ of the total TBI population, as reported by departmental statistics.

\section{Discussion of Results}

The clinical practice change by the nursing staff is supported by the structured implementation of the TBI protocol which in turn 


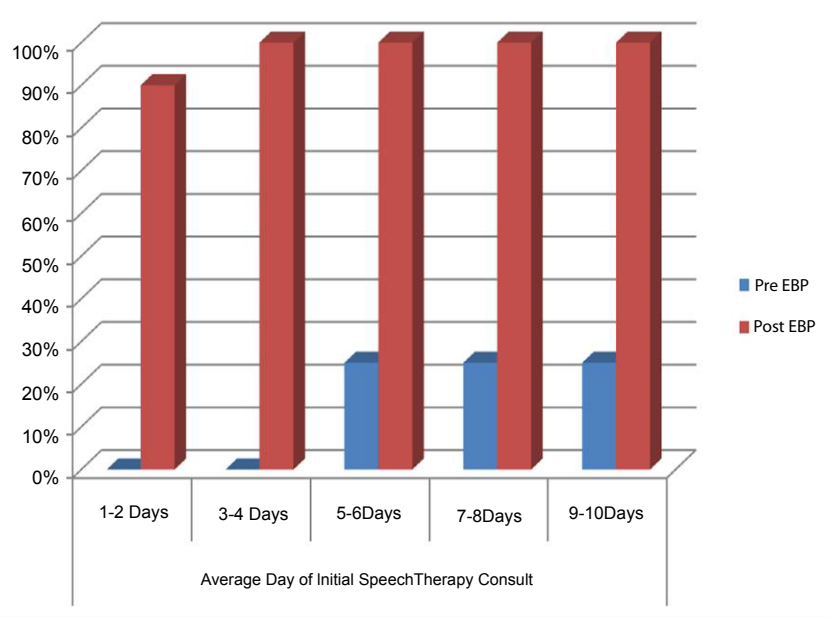

Figure 3: Speech Therapy Consults

became a template in care delivery for the TBI patient population. The protocol created a structured approach to clinical patient management for the nursing staff. The continuous repetition of patient interventions supported by the evidence-based protocols in concert with staff education on traumatic brain injury and its consequences, created skill development in the nursing staff for assessing and managing altered states in this patient population. Through the recognition of subtle changes in vital signs, the nursing staff was able to recognize that physiologic behavior and cognitive states become greatly altered, which in turn, present as states of confusion and agitation in the TBI patient. The daily cognitive evaluation together with the every-two hour vital signs enabled patient intervention to occur in a more focused and timely manner.

The timeliness of the ancillary services of physical therapy and speech/cognitive therapy also improved following the implementation of the TBI protocol. Prior to program implementation, physical therapy consults were consistently completed on day three or four from date of admission. The intensive physical therapy program provided the opportunity for patients to gait train and ambulate twice daily in the physical therapy department, and on an every two-hour basis on the nursing unit, which enhanced physical strength and independence and promoted regulation of sleep-wake cycles. Findings also support that the increase in timing stems from consistency of consult requests at time of admission as well as the recognition of TBI patient priority by the physical therapy department staff (Figure 2).

As for speech/cognitive therapy, the progressive increase may be attributed to the timely and consistent order entry for all TBI patients on admission, rather than the few patients eligible for post acute rehabilitation services. Another reason may be that protocol driven guidelines remove the inconsistency of primary provider discretion in requesting a consult. Through the implementation of the TBI protocol, patients received cognitive stimulation on a consistent basis which enabled patients to have the opportunity to strengthen verbal responses and develop an awareness that an event has occurred. Since project implementation, the speech/cognitive service had shown an increase to $100 \%$ for all TBI patients within 48 -hours of admission (Figure 3).

The finding for sitter session usage showed a reduction from 30 sessions 3 months before program implementation, to 6 sessions during project implementation, with continued sitter session reduction of 0 sessions for 6 months post program implementation. These findings are attributed to the efficacy in consistent adherence to an established protocol, which suggests that a change in clinical practice will occur over time.

\section{Future Research}

It is recommended that future research should be focused on whether a correlation exists between intensive cognitive therapy and improved $\mathrm{O}$-Log scores. It is also suggested that further research be undertaken to evaluate the effectiveness of early intensive therapy intervention on functional outcomes in TBI during the acute hospitalization, and the impact that early intensive therapy intervention has on length of stay. Future research is also recommended to correlate whether a reduction in sitter sessions was due to evidence-based practice protocol implementation versus a change in the severity of TBI admissions (Mild TBI: Glasgow Coma Scale of 13 or greater versus Moderate TBI: Glasgow Coma Scale of 12 or less).

\section{Implications for Practice}

The results of this program indicate that the EBP protocol created a structured approach to clinical patient management for the nursing staff. The continuous repetition of patient interventions supported by the protocols in concert with staff education on TBI and its consequences, created skill development in the nursing staff for assessing and managing altered states in this patient population. For continued sustainability of this program, integration of educational content on the TBI protocols with focused staff education on etiology, physiological and cognitive consequences of TBI were incorporated into the orientation of all new staff. The maintenance of program integrity was accomplished through the incorporation of clinical competencies in TBI management into yearly staff performance evaluations.

\section{Conclusions}

The integration of physical and cognitive interventions ultimately provided the patients with strategies to accomplish real-life demands and performance of everyday activities while in the acute care setting. Through the strategic cycle of assessment, ambulation and toileting, and hemodynamic status evaluation, patients became less apt to develop confused and agitated states thus supporting a safer patient environment. The integration of physical and cognitive interventions ultimately provided patients with strategies to accomplish real life demands and performance of everyday activities.

\section{References}

1. www.nunds.nih.gov

2. www.cdc.gov

3. Institute of Medicine (2001) Crossing the quality chasm: a new health system for the 21stcentury. National Academy Press, Washington.

4. Kay T, Harrington DE, Adams R, Anderson T, Berrol S, et al. (1993) Definition of mild traumatic brain injury. J Head Trauma Rehabil 8: 86-87.

5. Chapman EH, Weintraub RJ, Milburn MA, Pirozzi TO, Woo E (1999) Homeopathic treatment of mild traumatic brain injury: a randomized, doubleblind, placebo-controlled clinical trial. J Head Trauma Rehabil 14: 521-542.

6. Wade DT, Crawford S, Wenden FJ, King NS, Moss NE (1997) Does routine follow up after head injury help? A randomized controlled trial. J Neuro Neurosurg Psychiatry 62: 478-484.

7. Cifu DX, Kreutzer JS, Kolakowsky-Hayner SA, Marwitz JH, Englander J (2003) The relationship between therapy intensity and rehabilitative outcomes after traumatic brain injury: a multicenter analysis. Arch Phys Med Rehabil 84: 14411448.

8. Shiel A, Burn JP, Henry D, Clark J, Wilson BA, et al. (2001) The effects of 
Citation: Kutzleb J (2012) Evidence-Based Practice Nursing Interventions for Improved Functional and Cognitive Outcomes in the Traumatic Brain Injury Patient. - 11 XUK\&\&DH 1:110. doi:10.4172/2167-1168.1000110

increased rehabilitation therapy after brain injury: results of a prospective controlled trial. Clin Rehabil 15: 501-514.

9. Marion DW (2006) Evidenced-based guidelines for traumatic brain injuries. Prog Neurol Surg 19: 171-196.

10. Alderso AL, Novack TA (2002) Measuring recovery of orientation during acute rehabilitation for traumatic brain injury: value and expectations of recovery. $J$ Head Trauma Rehabil 17: 210-219.
11. Novack TA, Dowler RN, Bush BA, Glen T, Schneider JJ (2000) Validity of the Orientation Log, relative to the Galveston Orientation and Amnesia Test. J Head Trauma Rehabil 15: 957-961.

12. Titler MG, Kleiber C, Steelman VJ, Rakel BA, Budreau G, et al. (2001) The lowa Model of Evidence-Based Practice to Promote Quality Care. Crit Care Nurs Clin North Am 13: 497-509. 